(C) 2008 The Japan Society of Applied Physics

\title{
Variable Transverse-Electric and Transverse-Magnetic Mode Splitter Using Nickel Diffusion and Annealed Proton-Exchange Lithium Niobate Waveguides
}

\author{
Chin-Yu CHANG ${ }^{1}$, Cheng-Chih LAI ${ }^{2}$, and Way-Seen WANG ${ }^{1,2 *}$ \\ ${ }^{1}$ Department of Electrical Engineering, National Taiwan University, Taipei 10617, Taiwan, R.O.C. \\ ${ }^{2}$ Graduate Institute of Photonics and Optoelectronics, National Taiwan University, Taipei 10617, Taiwan, R.O.C.
}

(Received November 29, 2006; accepted October 11, 2007; published online January 18, 2008)

A novel variable transverse-electric and transverse-magnetic (TE-TM) mode splitter using nickel diffusion and annealed proton-exchange waveguides for the TE and TM branches of the splitter is presented. Due to the single-polarization property of the annealed proton-exchange waveguide, the measured polarization extinction ratio for the TE mode is higher than that reported. The measured polarization extinction ratio for the TM mode is as good as that reported, yet the fabrication process of single nickel diffusion is much simpler than that of double zinc-and-nickel diffusions. Moreover, the tunabilities of both TE and TM modes are found to be higher than those reported. [DOI: 10.1143/JJAP.47.179]

KEYWORDS: mode splitter, ridge waveguide, lithium niobate, nickel diffusion, annealed proton-exchange, $Y$-branch

\section{Introduction}

Transverse-electric and transverse-magnetic (TE-TM) mode (polarization) splitters are important optical devices for fiber communication systems and fiber sensors when orthogonal polarization states of signals are emphasized. For this purpose, various TE-TM mode splitters have been demonstrated. In principle, there are two methods for mode splitting. The first method uses optical interference in a directional coupler; thus, the TE and TM modes of the directional coupler can be split by the difference in phase velocity between the fundamental and first-order modes. ${ }^{1-4)}$ The directional coupler is advantageous owing to its economical use of a device area. However, the coupling lengths of the TE and TM modes must be carefully designed to achieve a high extinction ratio. This approach leads to a limited wavelength range of operation and rather stringent fabrication tolerances. The second method uses the mode sorting effect in an asymmetrical $Y$-junction waveguide; thus, the incident TE and TM modes are split by two waveguide branches owing to their different preferences of polarizations. ${ }^{5)}$ This type of mode splitter is more tolerant to fabrication errors and insensitive to wavelength variation. ${ }^{5-7)}$

In a conventional TE-TM mode splitter, the ratio of TE output power to TM output power is dependent on the polarization of the incident light, and the power ratio cannot be adjusted when the polarization of the incident light is fixed. However, in a practical application, waveguides are connected to fibers, fixed TE or TM output power ratios cannot be maintained owing to polarization rotation in fibers. Recently, a variable mode splitter has been reported, in which the power ratio of output waveguides can be electrically changed to provide a tuning mechanism for polarization splitting. ${ }^{8)}$ The device can then be used in a polarization-diversity receiver to equalize the power variation at different polarizations. However, the two output waveguides were fabricated by zinc-and-nickel diffusion at two close temperatures, 950 and $800{ }^{\circ} \mathrm{C}$, such that the effect of the second diffusion on the previously diffused waveguide must be carefully considered. Moreover, Liao and Lu have demonstrated a wide-angle TE-TM mode splitter consisting of a nickel-diffused waveguide and a proton-exchange (PE)

*E-mail address: wswang@cc.ee.ntu.edu.tw

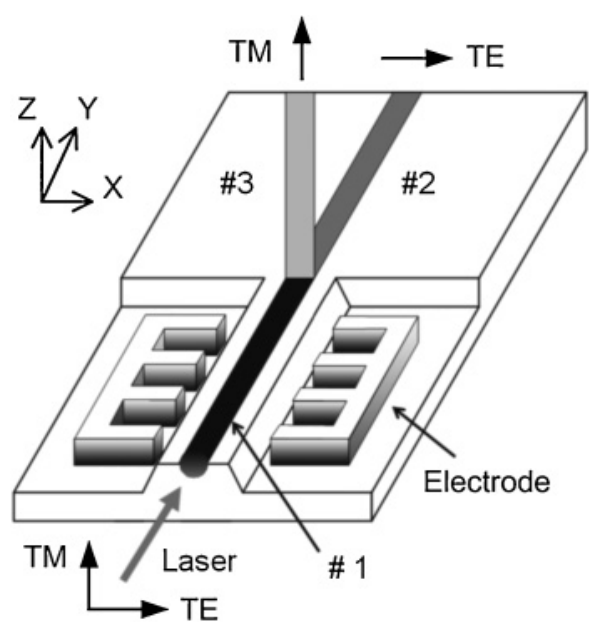

Fig. 1. Configuration of variable TE-TM mode splitter with ridge-type waveguide. Section \#1 is a nickel diffusion waveguide for both TE and TM waves, section \#2 is another nickel diffusion waveguide for a TE wave only, and section \#3 is an APE waveguide for a TM wave only.

waveguide. ${ }^{9)}$ Their basic idea of a wide angle is based on the use of a substrate prism in the PE waveguide for a TM wave. However, the PE waveguide has a higher propagation loss. This makes the device less useful. When the PE waveguide is replaced by an annealed proton-exchange (APE) waveguide for a lower propagation loss, the refractive index change of the APE waveguide is too small to have an efficient substrate prism for wide-angle bending.

In this work, we investigated an alternative method for the fabrication of the variable TE-TM mode splitter, as shown in Fig. 1. To minimize the effect of the second diffusion, the straight and bent waveguides were fabricated by nickel diffusion and annealed proton exchange, respectively. The fabrication of the nickel diffusion waveguide is simpler than that of the zinc-and-nickel diffusion waveguide, and the APE waveguide can be fabricated at a relatively lower temperature $\left(200^{\circ} \mathrm{C}\right)$, which has no significant effect on the existing nickel diffusion waveguide. Note that the TE mode is not supported in the APE waveguide and is left in the nickel diffusion waveguide based on a complete modesorting effect. Therefore, a relatively higher extinction ratio can easily be achieved. 


\section{Design Principle}

The proposed mode splitter is essentially an asymmetric $Y$-branch, consisting of three waveguides on a $Z$-cut $Y$ propagation $\mathrm{LiNbO}_{3}$ substrate. The input waveguide (\#1), fabricated by nickel diffusion, supports both TE and TM modes. As the polarization of the nickel diffusion waveguide is dependent on the choice of process parameters, ${ }^{10)}$ the TE-branch waveguide (\#2), supporting only TE-mode wave, can also be fabricated by nickel diffusion. The TM-branch waveguide (\#3), bent from the input waveguide at an angle of $0.5^{\circ}$ for the TM-mode wave, is fabricated by annealed proton exchange.

To become a variable mode splitter, a pair of comb-like electrodes is deposited on both sides of the input waveguide for TE-TM mode conversion. Thus, the TE and TM modes can be converted by applying an electric field along the $x$ direction owing to the off-diagonal electro optic coefficient $r_{51}$ of $\mathrm{LiNbO}_{3}$. The purpose of using a ridge structure is to increase the overlap integral between the applied electric field and the optical field.

\section{Fabrication}

To fabricate the proposed device, the ridge structure must be formed first, then the waveguides and electrodes. To fabricate the ridge structure, a silicon mask of $3000 \AA$ thickness was sputtered and patterned on the $\mathrm{LiNbO}_{3}$ substrate. The samples were then immersed in benzoic acid at $240^{\circ} \mathrm{C}$ for $6 \mathrm{~h}$ to form a proton-exchang layer. ${ }^{11)}$ However, the regions under the silicon mask were hardly affected. To reveal the ridge structure, the silicon mask and proton-exchang layer were removed using a mixture of HF and $\mathrm{HNO}_{3}$ (the volume ratio is $1: 2$ ) for $6 \mathrm{~h}$. The measured ridge height is $2 \mu \mathrm{m}$.

Then a nickel strip of $8 \mu \mathrm{m}$ width and $300 \AA$ thickness was deposited for the input and TE-branch waveguides. Another nickel strip of the same width but of a different thickness (400 $\AA$ ) was also deposited for the input waveguide. Note that the final thicknesses of nickel strips for the input and TE-branch waveguides are 700 and $300 \AA$, respectively. That is to use the process-dependent guiding property of nickel diffusion waveguides, ${ }^{10)}$ the sample was then wrapped with a $0.5 \mathrm{~mm}$ platinum foil and heated at $400^{\circ} \mathrm{C}$ for $1 \mathrm{~h}$ to oxidize the nickel strips, which is necessary for preventing the nickel strips from broadening due to the abrupt increase in temperature. Then the sample was diffused at $1000{ }^{\circ} \mathrm{C}$ for 90 min. Experimental results show that the out-diffusion effect was not significant under this process condition.
Table I. Fabrication techniques and process parameters for proposed splitter.

\begin{tabular}{llcc}
\hline \multicolumn{1}{c}{ Section } & Fabrication technology & $\begin{array}{c}\text { Temperature } \\
\left({ }^{\circ} \mathrm{C}\right)\end{array}$ & $\begin{array}{c}\text { Time } \\
(\mathrm{h})\end{array}$ \\
\hline Input & Ni $(700 \AA)$ diffusion & 1000 & 1.5 \\
TE branch & Ni (300 ̊) diffusion & 200 & 1.5 \\
\multirow{2}{*}{ TM branch } & Proton exchange & 350 & 0.5 \\
\hline
\end{tabular}

The next step is to fabricate the TM-branch waveguide by APE. The previous proton exchange was carried out again but at $200^{\circ} \mathrm{C}$ for $1.5 \mathrm{~h}$ and then annealing was performed at $350{ }^{\circ} \mathrm{C}$ for $30 \mathrm{~min}$. The fabrication process parameters of the waveguide branches are listed in Table I. Note that the APE process has no significant effect on the guiding property of the existing nickel diffusion waveguides because of the relatively lower temperature of the APE process.

Finally, a pair of periodic electrodes was fabricated onto the input section for TE-TM mode conversion. The electrode period $(\Lambda)$, electrode length $(L)$, and inter electrode gap $(d)$ were chosen to be $7 \mu \mathrm{m}, 5.6 \mathrm{~mm}$, and $15 \mu \mathrm{m}$, respectively. An aluminum layer of $4000 \AA$ thickness was deposited by thermal evaporation and patterned by standard photolithography. The sample was then etched in pure phosphoric acid at $60^{\circ} \mathrm{C}$ for $35 \mathrm{~s}$ to obtain the periodic electrodes.

\section{Experimental Results}

The fabricated TE-TM mode splitter was characterized using the measurement setup shown in Fig. 2. First, the sample was end-coupled with a He-Ne laser of $0.6328 \mu \mathrm{m}$ wavelength $(\lambda)$, and the output intensity contours were captured using a charge-coupled-device (CCD) camera. The measured propagation losses are 1.1 and $2 \mathrm{~dB} / \mathrm{cm}$ for the TE and TM modes of the nickel diffusion waveguide, and $0.9 \mathrm{~dB} / \mathrm{cm}$ for the annealed proton-exchange waveguide. Figures 3(a)-3(c) show the output intensity contour profiles when the incident light is polarized at angles of $0^{\circ}$ (TM wave), $90^{\circ}$ (TE wave), and $45^{\circ}$ (both TE and TM waves) relative to the $z$-axis, respectively. Note that the vertical positions of the output profiles are different because the waveguides were fabricated by two different processes, APE and nickel diffusion. The waveguide depths of APE and nickel diffusion estimated from the figures are 1.4 and $2.5 \mu \mathrm{m}$, respectively. The waveguides were verified experimentally to support only single modes.

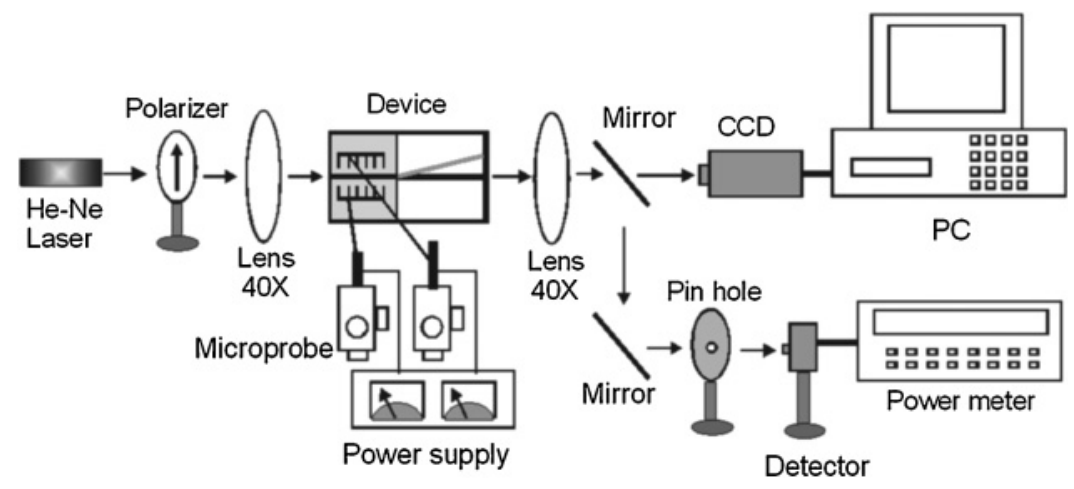

Fig. 2. Measurement setup. 

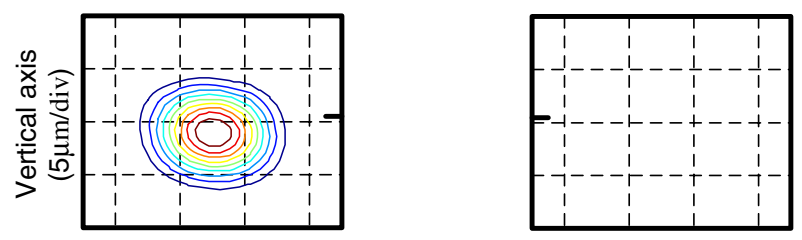

(a)
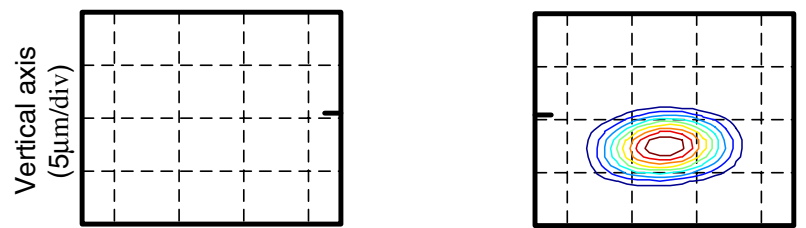

(b)

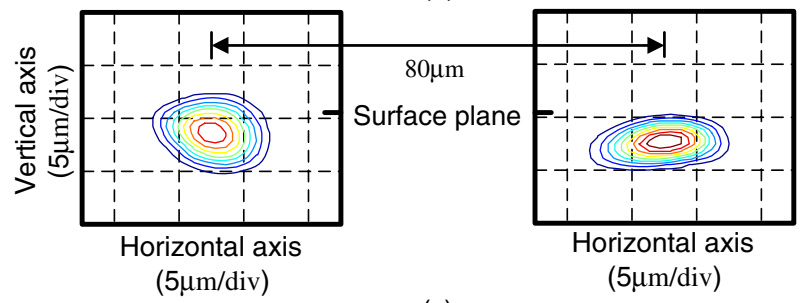

(c)

Fig. 3. (Color online) Output near field intensity contour profiles for different incident waves: (a) TM wave only, (b) TE wave only, and (c) both TM and TE waves.

For comparison, the extinction ratios of the TE and TM modes were defined as

$$
\begin{aligned}
\xi_{\mathrm{TE}} & =10 \log \left(P_{\mathrm{TE}, \mathrm{TE}-\text { branch }} / P_{\mathrm{TE}, \mathrm{TM}-\text { branch }}\right) \\
\xi_{\mathrm{TM}} & =10 \log \left(P_{\mathrm{TM}, \mathrm{TM}-\text { branch }} / P_{\mathrm{TM}, \mathrm{TE}-\text { branch }}\right),
\end{aligned}
$$

where $P_{\mathrm{TE}, \mathrm{TE}-b r a n c h}$ is the TE output power in the TEbranch waveguide, which is similar to the definitions for $P_{\mathrm{TE}, \mathrm{TM}-\text { branch }}, P_{\mathrm{TM}, \mathrm{TM}-\text { branch }}$, and $P_{\mathrm{TM}, \mathrm{TE}-\text { branch }}$. The measured $\xi_{\mathrm{TE}}$ is $25 \mathrm{~dB}$, which is higher than that reported. ${ }^{8)}$ Because the APE waveguide supports only singly a TM-polarized wave, the unwanted TE components of an incident wave are not allowed in the APE waveguide, and therefore, they are left in the nickel diffusion waveguide. The measured $\xi_{\mathrm{TM}}$ is $18 \mathrm{~dB}$, which, just as expected, is the same as that reported. ${ }^{8)}$ However, as only one cycle of nickel diffusion is required, the fabrication process is much simpler than that in two cycles of zinc-and-nickel diffusion. ${ }^{8)}$

Then, a voltage was applied to the mode converter. The normalized TE and TM output powers versus the applied voltage were measured with the incident light polarized at $45^{\circ}$. For comparison, the tunabilities (tuning efficiencies) for the TE and TM modes were defined as

$$
\begin{aligned}
& \eta_{\mathrm{TE} \rightarrow \mathrm{TM}}=\left.\frac{P_{\mathrm{TM}}(\mathrm{V})-P_{\mathrm{TM}}(0)}{P_{\mathrm{TM}}(0)}\right|_{\mathrm{TM}-\text { branch }}, \\
& \eta_{\mathrm{TM} \rightarrow \mathrm{TE}}=\left.\frac{P_{\mathrm{TE}}(\mathrm{V})-P_{\mathrm{TE}}(0)}{P_{\mathrm{TE}}(0)}\right|_{\mathrm{TE}-\text { branch }},
\end{aligned}
$$

where $P_{\mathrm{TE}}$ and $P_{\mathrm{TM}}$ are the voltage-dependent output powers of the TE- and TM-branch waveguides. Note that the tunabilities include the TE-TM mode conversion and mode splitting efficiencies. The measured tunabilities are shown in Fig. 4. As can be observed from the figure, when a voltage of $70 \mathrm{~V}$ is applied to the periodical electrodes, 85 and $50 \%$ of the powers in the TM- and TE-branch waveguides are

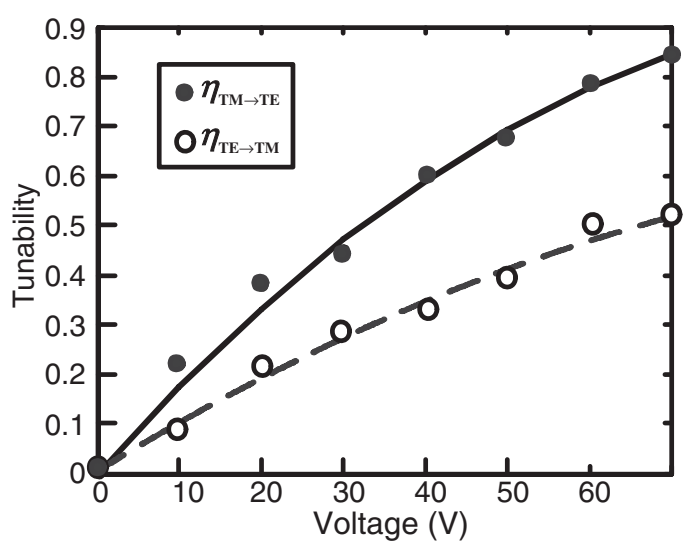

Fig. 4. Tunability vs applied voltage.

converted and directed to the TE- and TM-branch waveguides, respectively. As the propagation losses are polarization dependent, the conversion efficiencies for the TE and TM modes are different. These can be further tailored by fine process control to have an equal or a certain ratio for different of applications.

\section{Discussion}

The proposed variable TE-TM mode splitter consists of a mode converter and a mode splitter. In refs. 5-7, Goto and coworkers have shown that a mode splitter based on a $Y$ junction is less wavelength dependent. However, the mode converter is wavelength sensitive. The optimum condition for polarization control is strongly affected by the electrode gap length of the comb like electrode, the effective indices of the waveguide modes, and the light wavelength. For example, we have tried using four sets of comb like electrodes; the periods of the electrodes are 7, 8, 9, and $10 \mu \mathrm{m}$. These four sets of electrode periods are slightly different from the theoretically predicted one $(8.5 \mu \mathrm{m})$. The electrode period that gives rise to the best results is used for the proposed mode splitter. This indicates that the proposed variable TE-TM mode splitter is wavelength sensitive, though the mode splitting part of the device is wavelength insensitive.

Initially, the measured voltage for the polarization control in the fabricated device seems to be relatively large. This can be understood by comparing with the planar-type (without the ridge) mode splitter. When $d=5 \mu \mathrm{m}$ and $L=6 \mathrm{~mm}$ are assumed at $\lambda=0.5995 \mu \mathrm{m}$, a tuning voltage of $9 \mathrm{~V}$ is required. ${ }^{12)}$ The calculated tunability is $40 \%$. In principle, the tuning voltage, to the first order, is proportional to $\lambda d / \gamma L$. When the overlap integral $\gamma$ is assumed to be the same, the voltage for the same tunability in our case ( $\lambda=0.6328 \mu \mathrm{m}, d=15 \mu \mathrm{m}$, and $L=5.6 \mathrm{~mm}$ ) is estimated to be $31 \mathrm{~V}$. Actually, only $24 \mathrm{~V}$ is applied. This is due to the increase in overlap integral when the ridge structure is used. The tuning voltage is reduced by a factor of about $1 / 3$, which is the same as that reported previously. ${ }^{8)}$ Nevertheless, the present tunabilities are higher than those reported. ${ }^{8)}$ Further improvement of the fine adjustment of the TE and TM tunabilities using a proper design of the electrodes should be an interesting future work. 


\section{Conclusions}

A variable TE-TM mode splitter using nickel diffusion and annealed proton exchange for the TE and TM branches of the splitter is successfully demonstrated. Experimental results show that the measured extinction ratio for the TE mode is $25 \mathrm{~dB}$, which is higher than that reported due to the single-polarization property of the APE waveguide, and the measured extinction ratio for the TM mode is $18 \mathrm{~dB}$, which is as good as that reported, yet the fabrication process is much simpler. Details of the application and improvement of the devices will be of interest in the future.

\section{Acknowledgement}

This work was supported by the National Science Council, Taipei, Taiwan, under contract No. NSC-952221-E-002-383.
1) O. Mikami: Appl. Phys. Lett. 36 (1980) 491

2) D. Yap, L. M. Johnson, and G. W. Pratt, Jr.: Appl. Phys. Lett. 44 (1984) 583.

3) H. Maruyama, M. Harana, and H. Nishihara: J. Lightwave Technol. 13 (1995) 1550

4) R. C. Twu, C. C. Huang, and W. S. Wang: Electron. Lett. 36 (2000) 220.

5) N. Goto and G. L. Yip: J. Lightwave Technol. 7 (1989) 1567.

6) J. J. G. M. van der Tol and J. H. Laarhuis: J. Lightwave Technol. 9 (1991) 879 .

7) J. J. G. M. van der Tol, J. W. Pedersen, E. G. Metaal, Y. S. Oei, H. van Brug, and I. Moerman: IEEE Photonics Technol. Lett. 5 (1993) 1412.

8) W. H. Hsu, K. C. Lin, J. Y. Li, Y. S. Wu, and W. S. Wang: IEEE J. Sel. Top. Quantum Electron. 11 (2005) 271.

9) Y.-P. Liao and R.-C. Lu: IEEE J. Sel. Top. Quantum Electron. 6 (2000) 88

10) Y.-P. Liao, D.-J. Chen, R.-C. Lu, and W.-S. Wang: IEEE Photonics Technol. Lett. 8 (1996) 548.

11) F. Laurell, J. Webjorn, G. Arviddson, and J. Homberg: J. Lightwave Technol. 10 (1992) 1606.

12) R. C. Alferness: Appl. Phys. Lett. 36 (1980) 513. 